\title{
OTTO TOEPLITZ: ALGEBRAIKER DER UNENDLICHEN MATRIZEN
}

\author{
STEFAN MÜLLER-STACH
}

\begin{abstract}
Zusammenfassung. Otto Toeplitz ist ein Mathematiker, dessen Schicksal exemplarisch für die Vernichtung der jüdischen wissenschaftlichen Elite in Deutschland durch die Nationalsozialisten ist. Sein Einfluss in der Mathematik ist noch heute, besonders durch den Begriff der Toeplitzmatrizen, deutlich spürbar. Die kulturgeschichtliche Bedeutung von Toeplitz ist ebenso groß. Als Gründungsherausgeber von zwei Zeitschriften hat er sein Engagement für die Didaktik und die Wissenschaftsgeschichte untermauert. Wir geben einen Einblick in sein Schicksal und seine Gedankenwelt unter dem Einfluss von David Hilbert und Felix Klein.
\end{abstract}

\section{EINLEITUNG}

Die Mathematischen Semesterberichte wurden 1932 von Heinrich Behnke und Otto Toeplitz gegründet. Heinrich Behnke mit seinen herausragenden Verdiensten um die Mathematikdidaktik, die Lehrerfortbildung, seinem mathematischen (Euvre und seiner in der Nachkriegszeit so einflussreichen Schule ist im Hinblick auf die Semesterberichte schon gewürdigt worden [10, 11]. Mit dem vorliegenden Aufsatz wollen wir nun stattdessen einmal Otto Toeplitz gerecht werden.

Es soll insbesondere herausgearbeitet werden, wie nahe die Idee der Semesterberichte zur Gedankenwelt von Toeplitz und der von Behnke ist. Toeplitz, der aus Breslau stammte, wurde zunächst in Göttingen von David Hilbert und Felix Klein stark beeinflusst. Ab den 1920er Jahren entwickelte er aber vollkommen eigene Ideen, sowohl in seiner Forschung als auch im Bereich der Wissenschaftsgeschichte und der Hochschuldidaktik. Die beiden von ihm gegründeten Zeitschriften, seine historischen Seminare sowie seine Reden und Schriften zeigen seine Individualität und seine mittlerweile erreichte inhaltliche Distanz zu seinen beiden Vorbildern, mit denen er auch eine umfangreiche Korrespondenz unterhielt.

Für Toeplitz bedeutete Deutschland die Erfüllung seiner wissenschaftlichen Träume. Durch die Nationalsozialisten wurde Toeplitz aus seinem Amt entfernt und emigrierte 1939 nach Jerusalem, wo er Anfang 1940 starb. Zwischen 1933 und 1939 hatte er weiter intensiv seine Ideen verfolgt und sich für die Juden in Deutschland in mehrfacher Weise eingesetzt.

\section{Otto Toeplitz: Der Mensch}

Otto Toeplitz muss eine bemerkenswerte Persönlichkeit mit herausragenden Charaktereigenschaften gewesen sein. Dies wird wunderbar in der liebevollen Beschreibung aus einem Nachruf von Heinrich Behnke [3, S. 1] ausgedrückt:

Otto Toeplitz war Mathematiker. Aber das charakterisiert ihn bei weitem nicht. Er war zugleich Pädagoge und Historiker seines Faches und zu allererst ein hilfsbereiter, mitfühlender Mensch. Er gehörte zu den Charakteren, deren Bild man nicht

Erweiterte Fassung eines Vortrags zum 80jährigen Bestehen der Mathematischen Semesterberichte, der am 23. April 2013 in Bonn gehalten wurde. 
wieder aus dem Gedächtnis verliert, wenn man mit Ihnen auch nur einmal ein Gespräch geführt hat. Er lauschte den Worten seines Gegenübers, wer auch immer es war, mit ganz ungeteilter Aufmerksamkeit - wie es so selten geschieht - und sprach dann selbst mit großem psychologischen Einfühlungsvermögen. Seine freundlichen Augen, sein Mienenspiel, all seine Bewegungen standen im Banne der geistigen Vorgänge in ihm. Er sprach mit einer wohltuenden Wärme, einer Offenheit und einer Kritik an sich selbst, die immer wieder überraschte ...

Aus allem, was er tat, sprach sein Verantwortungsgefühl gegenüber den geistigen Mächten, seine Liebe zu Deutschland, vor allem zur akademischen Jugend, und seine Verpflichtung gegenüber der deutschen Universität, - nicht zufällig hat Karl Jaspers die 1. Auflage seines Buches „Die Idee der Universität” ihm gewidmet.

Die darin beschriebene Liebe zu Deutschland, und das Engagement von Otto Toeplitz als Universitätsprofessor wurden schmerzlich verraten durch das Schicksal, das ihm nach 1933 zusammen mit den anderen jüdischen Kollegen widerfuhr [1, S. 103]. Weitere Nachrufe und Beschreibungen der Person von Otto Toeplitz verfassten Stefan Hildebrandt [5] und Wilhelm Klingenberg [4]. Nachrufe zum wissenschaftlichen Werk erschienen von Peter Lax in [5], Jean Dieudonné in [8], Israel Gohberg in 8] und Gottfried Köthe in [8] sowie [15. Die Kinder von Otto und Erna Toeplitz, die Tochter Eva Wohl [52] und der Sohn Dr. Uri Toeplitz [51, haben Autobiographien geschrieben, in denen ihre Eltern eine wichtige Rolle spielen. Eva Wohl bestätigt die Einschätzung von Behnke in ihren Erinnerungen „So einfach liegen die Dinge nicht" (eine Redensart von Otto Toeplitz) [52, S. 40]:

Um seine Vorlesungen vorzubereiten, machte Vater oft einen Spaziergang durch die Kaiser-Friedrich-Straße bis ans Rheinufer. Manchmal durfte ich ihn dabei begleiten. Dann hielt er mich an seiner Hand und ließ mich hopsen. Dabei prüfte er mich auf mathematische Scherzfragen, von denen eine war: Zwei Väter und zwei Söhne aßen zusammen drei Eier, wie ist das möglich? Er hatte ein ganzes Buch solcher Fragen, sie stammten aus dem Besitz seines Vaters. Ein anderer Scherz war die Gleichung $(\mathrm{Klim}+\mathrm{Bim})^{2}=\mathrm{Klim}^{2}+2 \mathrm{Klimbim}+\mathrm{Bim}^{2} \ldots$ Mein Vater war ein sehr attraktiver Mann. Es muss wohl sein außerordentlicher Charme gewesen sein, der ihn so beliebt bei Frauen machte. Er konnte sich leicht in die Lage seines Gegenübers versetzen und war deshalb interessant in der Unterhaltung. Eine seiner Maximen war: „Man muss hinhören können". Zudem war er äußerst gebildet und interessiert an vielen Dingen, auch solchen, die außerhalb der Mathematik lagen. Wie ich schon erwähnt habe, liebte er Musik, und auch die leiblichen Genüsse, gutes Essen und Trinken, waren ihm sehr wichtig. Dazu war er einer der gütigsten Menschen, die man sich vorstellen kann.

Das Buch von Eva Wohl berichtet ausführlich über die Kindheit in Deutschland und ihr Leben nach ihrer Auswanderung im Jahre 1939. Dr. Erich (Uri) Toeplitz war Flötist und Mitbegründer des Israel Philharmonic Orchestra, das seit 1936 bestand. Er schreibt über seinen Vater in [51, S. 49]:

Von Zeit zu Zeit kamen Mathematiker nach Kiel zu Besuch. Sie wurden dann gastlich aufgenommen und wohnten auch bei uns. Häufiger Gast war Ernst Hellinger, Professor in Frankfurt am Main, ein alter Freund und Studienfreund meines Vaters aus Breslau ... Hellinger spendierte zum Nachmittagskaffee regelmäßig die schönsten Stücke Kuchen, die wir Kinder aussuchen und einkaufen durften ... Eines Tages kamen in Kiel zwei „Wandervögel” zu uns, beide in kurzen Hosen, Professoren der Mathematik, welche die Ostsee entlang gewandert waren. Es waren Reidemeister 
aus Königsberg und Rademacher aus Breslau. Mit dem letzteren entwickelte sich eine nähere Beziehung. Beide, Vater und er, hielten an ihren Universitäten Vorlesungen "für Hörer aller Fakultäten", in denen sie versuchten, die Mathematik für Laien verständlich darzustellen ... Rademacher schlug vor, die besten dieser Vorlesungen auszusuchen, und in Buchform zu veröffentlichen. Vater stimmte zu, obwohl er dies lieber alleine gemacht hätte, und „Von Zahlen und Figuren, Proben mathematischen Denkens für Liebhaber der Mathematik", angefangen in Kiel, erschien im Jahr 1930 ... Schon wenn man das Vorwort liest, erkennt man den persönlichen Stempel meines Vaters, sagen wir, seinen wunderbar musikalischen Stil. Während der Arbeit kam es häufig vor, daß sich die Autoren nicht einig waren, ob ein Kapitel wirklich allgemeinverständlich sei. Da wurde ich eingeschaltet.

Uri Toeplitz starb 2006. Auch sein Sohn Gideon war Musiker und leitete viele Jahre lang als Manager das Pittsburg Symphony Orchestra. Otto Toeplitz selbst hat seine Musikalität auch in seinen mathematischen Werken ausgedrückt, wie Uri Toeplitz und auch Eva Wohl beide betonen.

Max Born, der spätere Physik-Nobelpreisträger, schrieb 1940 einen Nachruf auf Otto Toeplitz in Nature [7. Die algebraische Seite von Toeplitz in der Tradition von Hilbert bringt er darin deutlich zum Ausdruck:

Professor Otto Toeplitz, who died on March 15 in Jerusalem, was one of that rare type of scholars who combine with a high standard of specialized research a deep interest in the history of their science and its bearing on general questions ... Toeplitz's mathematical interest was wide and covered all branches of research, but was deeply rooted in algebra. He liked to consider analysis as an algebra of an infinite number of variables, an example of which was given by Hilbert's treatment of integral equations as special cases of linear equations and quadratic forms of infinite variables.

Toeplitz lebte auch fort in seinen zahlreichen Schülern: Charlotte Schroeckh (Kiel 1917), August Prüss (Kiel 1918), Ulrich John (Kiel 1922), Hans Hansen (Kiel 1923), Robert Schmidt (Kiel 1923), Anna Stein (Kiel 1927), Walter Stein (Kiel 1930), Helmut Ulm (Bonn 1933), Anton Weber (Bonn 1934), Marie-Luise Schluckebier (Bonn 1935), Hans Schwerdtfeger (Bonn 1935), Dora Reimann (Bonn 1935), Arthur Donald Steele (Bonn 1936), Elisabeth Hagemann (Bonn 1937) und Bruno Ritzdorff (Bonn 1938). Diese Liste mit bemerkenswert vielen Frauen ist vermutlich unvollständig. Sie wurde mit Hilfe des Doktorandenarchivs von Renate Tobies bei der DMV (http://dmv.mathematik.de) und der Schülerkartothek von Toeplitz und Bessel-Hagen [19, S. 403] erstellt.

\section{Breslau, Kiel und Bonn: Biographisches}

Toeplitz wurde am 1. August 1881 in Breslau geboren und wuchs dort auf. Sein Vater Emil, und sein Großvater Julius waren beide Mathematiklehrer. Seine Mutter Pauline (eine Cousine von Emil) starb an Tuberkulose als Toeplitz 11 Jahre alt war. Er wuchs in strenger Erziehung als Einzelkind im Haushalt des Vaters Emil auf, der am gleichen Johannes Gymnasium in Breslau Lehrer war, auf das Toeplitz ging.

Toeplitz studierte dann auch in Breslau Mathematik. Sein Doktorvater war Jakob Rosanes und Toeplitz promovierte 1905 mit der Dissertation „Über Systeme von Formen, deren Funktionaldeterminante identisch verschwindet" im Gebiet der algebraischen Geometrie. Lebenslange Freundschaften verbanden Toeplitz aus der 
Breslauer Zeit mit Max Born, Richard Courant und Ernst Hellinger.

1906 wechselte Toeplitz nach Göttingen. Dort wurde er Schüler und Mitarbeiter von David Hilbert, der zu dieser Zeit über Integralgleichungen arbeitete, einem seiner Schwerpunkte nach den Arbeiten zur Invariantentheorie, zu den Grundlagen der Geometrie und zur Zahlentheorie. Otto Toeplitz setzte Hilbert ein Denkmal mit seinem Aufsatz „Der Algebraiker Hilbert” [40]. In diesem Artikel setzt sich Toeplitz mit dem Werk Hilberts auseinander und stellt interessanterweise seine eigene Forschung in eine historische Tradition mit Hilberts algebraischen Arbeiten. Darüber berichten wir weiter unten. Das Verhältnis von Hilbert und Toeplitz war sehr freundschaftlich, die Familien kannten sich und es gab auch in den Folgejahren noch Briefwechsel [44, 48. Franz Hilbert, der Sohn von David und Käthe Hilbert, erhielt offenbar Nachhilfe von Toeplitz (ebenso übrigens von Courant [21, S. 20]), wie aus einer Postkarte aus dem Nachlass [44] hervorgeht.

Ein enges Verhältnis existierte auch zwischen Felix Klein und Otto Toeplitz. Dabei drehte es sich unter anderem um Fragen der Hochschuldidaktik. Klein beschäftigte sich schon seit Ende des 19. Jahrhunderts mit didaktischen Fragen und der Reform der Schulen [18. Kleins Stil und Ideenwelt mit ihren konkreten Objekten unterschied sich deutlich von der abstrakteren Welt von Hilbert. Hierzu ein Zitat von Behnke [3, S. 3];

Aber Toeplitz' Wirken ist ganz und gar nicht auf diese Forschung beschränkt. Dazu war er ein zu vielseitiger Geist, und in Göttingen hat ihn nicht nur Hilbert beeinflußt. Felix Klein war ihm auch ein eindrucksvolles Vorbild und hat ihn veranlaßt, sich auch frühzeitig mit Fragen des Unterrichts zu befassen. Klein scheint dem jungen Göttinger Dozenten schon früh viel zugetraut zu haben. Er sprach mit ihm viel über die Didaktik der Anfängervorlesung, und wir können uns lebhaft vorstellen, wie Toeplitz den Geist der Hilbertschen Schule den klassisch-anschaulichen Auffassungen von Klein (der ja nicht einmal den Begriff der abstrakten Gruppe zulassen wollte) gegenüber verteidigte.

In der Göttinger Zeit war Toeplitz auch in Kontakt mit Hermann Minkowski 3, S. 1] und Hermann Weyl [21, S. 25], die Distanz zu beiden war aber größer als zu Hilbert und Klein. Im Jahr 1913 wechselte Toeplitz als außerordentlicher Professor nach Kiel. Aufgrund von Verzögerungen wurde er erst einige Jahre später, wie versprochen, Ordinarius als Nachfolger von Leo Pochhammer. Zeitweise war Toeplitz Dekan in Kiel und maßgeblich verantwortlich für einige Berufungen. In seiner Kieler Zeit waren auch Helmut Hasse als Privatdozent sowie Julius Stenzel, Heinrich Scholz und Ernst Steinitz in Kiel. Ein Versuch, Karl Jaspers aus Heidelberg nach Kiel zu holen, scheiterte. Anscheinend hatte es dabei eine antisemitische Reaktion innerhalb der Fakultät gegeben, die auf die jüdische Frau von Jaspers zielte, siehe den Beitrag von Uri Toeplitz in [4, S. 28]. Jaspers und Toeplitz führten einen Briefwechsel, der heute in Marbach untergebracht ist, ebenso existiert ein umfangreicher Briefwechsel zwischen Heinrich Behnke und Karl Jaspers [12]. Jaspers widmete Toeplitz die erste Auflage seines Buches „Die Idee der Universität”. Toeplitz arbeitete mit Ernst Hellinger mehrere Jahre an dem langen Übersichtsband [36] über Integralgleichungen, der in der Enzyklopädie der mathematischen Wissenschaften erschien. Über das Verhältnis zu Ernst Hellinger schreibt Uri Toeplitz [51, S. 49]: 
„Onkel Henga”, wie wir Kinder ihn nannten, war Junggeselle und besonders nett zu uns Kindern. Wir störten aber z.B., wenn die Erwachsenen sich bei Tisch unterhalten wollten. So setzte er uns ein Schweigegeld aus, und das klappte ... Hellinger spendierte zum Nachmittagskaffee regelmäßig die schönsten Stücke Kuchen, die wir Kinder aussuchen und einkaufen durften; das gab es bei uns nur, wenn er da war. Später, zwischen 1934 und 1936, als ich in Frankfurt lebte, hat er mich regelmäßig zum Mittagessen eingeladen.

Ernst Hellinger veranstaltete zusammen mit Max Dehn, Carl Ludwig Siegel und Paul Epstein ein historisches Seminar in Frankfurt [28]. Toeplitz hatte durch ihn somit Beziehungen zu diesem Frankfurter Seminar, wie sie auch bei Behnke [3, S. 4] erwähnt werden. Hellinger verbrachte einige Zeit in einem Konzentrationslager, bis er in die USA auswandern konnte. Er bekam 1939 eine Stelle als Lecturer an der Northwestern University in Evanston bei Chicago und starb dort 1950 als emeritierter Professor.

In dieser Kieler Zeit begann auch Toeplitz Einsatz für die Lehrerausbildung [41. Dazu richtete er ein mathematisch-didaktisches Kolloquium ein [10, S. 170] und kümmerte sich sowohl um die Fortbildung der Lehrer aus dem Umland und als auch um die Ausbildung der Referendare. Bei diesen Aktivitäten ist natürlich der starke Einfluss von Felix Klein zu erwähnen, der die sogenannte Kleinsche Reform des Unterrichts und die Ferienkurse für Lehrer begründet hatte. Einige didaktische Einsichten aus diesen Veranstaltungen publizierte Toeplitz in den ersten Ausgaben der Semesterberichte, in denen noch Unterrichtskonzepte für Lehrer behandelt wurden. So besprach er dort zum Beispiel „eingekleidete Gleichungen” oder die „Erneuerung" der Schulgeometrie [5, S. 60-62].

In Kiel hatte Toeplitz auch intensive Kontakte zu Heinrich Scholz und Julius Stenzel, siehe [3, S. 4]. Zusammen wurden Seminare zur Mathematik der Griechen abgehalten. Mehrere Jahre später in der Bonner Zeit gründete Toeplitz zusammen mit Julius Stenzel und Otto Neugebauer die Zeitschrift „Quellen und Studien zur Geschichte der Mathematik, Astronomie und Physik" [20]. Köthe [4, S. 14] berichtet über Wolfgang Franz, der das Kieler Seminar besuchte, und dort eine Diskussion über das fehlende Konzept von Irrationalzahlen bei den Griechen miterlebte.

Im Jahr 1927 erging an Toeplitz der Ruf nach Bonn. Als Nachfolger von Eduard Study trat er die Stelle im Sommersemester 1928 an. Toeplitz und seine Familie erlebten einen Aufschwung durch das Ankommen in Bonn, Uri Toeplitz nennt den Ruf sogar eine „Erlösung” nach den Kieler Jahren [4, S. 28], die für die Familie wohl durch Provinzialität und schlechte Erlebnisse, wie der Episode mit Jaspers Frau, geprägt waren, und betont auch an anderer Stelle [51, S. 54], dass ihm die rheinische Lebensart mehr entgegenkam.

Im Jahr 1930 erschien das Buch „Von Zahlen und Figuren” 35 von Hans Rademacher und Otto Toeplitz, das in der Kieler Zeit begonnen wurde. Es ist in viele Sprachen übersetzt worden. Max Born schreibt darüber in [7]:

But Toeplitz's general attitude to mathematics, which he preferred to consider more as an art than as a science, is more clearly visible in his quasi-popular book "Von Zahlen und Figuren" written with Rademacher in 1930, which seems to me a masterpiece of that class of scientific literature which attempts to instruct a wider public 
in the fundamental ideas of science. It is not easy, but certainly fascinating reading.

Heinrich Behnke bemerkt zu diesem Buch in [3, S. 6]:

Jede der 30 Nummern dieses Buches ist in ihrer Abgeschlossenheit und Abgewogenheit ein Kunstwerk für sich. Alles war in Vorträgen vor einem größeren Publikum auf seine Eignung durchgeprobt. So ist dieses kleine Büchlein viel mehr als es scheint, und bildet eine glänzende Einführung in heutige mathematische Probleme für solche Leser, die, ohne die Zeit aufzubringen, umfangreiche mathematische Theorien zu erlernen, in das Wesen der Mathematik eindringen wollen. Alle anderen populären Werke der letzten Jahrzehnte übertrifft es weit an pädagogischem Geschick und an sicherer Zielsetzung. Die ganze Darstellung wirkt wie ein Ausschnitt aus klassischen Werken.

Wie in [5, S. 22] berichtet wird, zeigte Felix Hausdorff bereits im Vorfeld der Berufung großes Interesse an Toeplitz und es gab einen sehr freundschaftlichen Kontakt zur Familie Toeplitz. Uri Toeplitz schreibt in [4, S. 26]:

Unsere Familien waren befreundet. Er war sehr vielseitig und hat, unter anderem, ein viel aufgeführtes Lustspiel geschrieben. Er war auch sehr musikalisch, und ich durfte mit ihm vierhändig spielen.

Israel Gohberg berichtet in [4, S. 34] über die mathematische Zusammenarbeit von Hausdorff und Toeplitz über das Spektrum von Operatoren, was sich vermutlich auf das sogenannte Toeplitz-Hausdorff Theorem bezieht.

Toeplitz entwickelte in dieser Zeit auch sein Interesse in Mathematikgeschichte in Zusammenarbeit mit Erich Bessel-Hagen und Oskar Becker weiter. Mit seinem Schüler Gottfried Köthe bekam er neue Ideen in der Forschung [42. Die hohen Studentenzahlen in Bonn veranlassten ihn, weitere Ideen in der Hochschuldidaktik der Anfängervorlesungen zu entwickeln. Aus diesen Quellen speiste sich auch seine „genetischen Methode”, die in einem Buchmanuskript 34 zu einer Anfängervorlesung zur Infinitesimalrechnung umgesetzt wurde, welches posthum Gottfried Köthe herausgab. In den folgenden Abschnitten berichten wir ausführlicher über die historischen und didaktischen Ansätze von Toeplitz sowie seine mathematischen Arbeiten.

\section{Die Zeit in Deutschland NaCh 1933 und in JeRusalem}

Ab 1933 wurden Juden mit Verweis auf das „Gesetz zur Wiederherstellung des Berufsbeamtentums" aus dem Beamtendienst entfernt. Toeplitz konnte noch bis 1935 vor den „Nürnberger Gesetzen” im Amt bleiben, da er bereits vor dem 1. Weltkrieg Beamter war, verlor aber die Prüfungsberechtigung. Otto Toeplitz drückte seine Gefühle über die Zeit kurz nach 1933 in einem Brief vom 22. Januar 1934 an David Hilbert aus [48:

... und da Klein nicht mehr ist, werden Sie der gewichtigste Zeuge von diesem Stück Geschichte sein, das Sie durch 35 Jahre miterlebt haben. Es war mir bisher vergönnt, dass ich meine mathematische Arbeit fortsetzen konnte. Meine Schüler sind mir treu geblieben und arbeiten weiter mit mir mit, soweit der Dienst ihnen irgend Zeit dazu lässt. Ich glaube, diese jungen Menschen kämpfen um den Ausgleich ihrer Pflichten und Ziele nicht minder, als es diese Zeit auch mir auferlegt, widerstreitende Empindungen in mir zu balancieren. Früher habe ich stets versucht, 
alles, was deutsch und alles, was jüdisch in mir war, hunderprozentig zu bejahen und zu einer fruchtbaren Synthese mit einander zu bringen; und ich habe an der Möglichkeit davon nie gezweifelt und sehe auch heute keinen Grund, meine Ansicht darüber zu ändern. Auch heute verleugne ich nichts von dem, was in mir deutsch ist und nichts von dem, was in mir jüdisch ist. Aber heute ist statt der Synthese der Kampf der beiden Momente gegen einander getreten, und das ist es, was soviel von den Kräften auffrisst, die man gerne einem besseren Zweck widmen möchte.

Uri Toeplitz beschreibt in [4, S. 28] auch diese Jahre. Nach dem erfolgreichen Ruf und dem Eintreffen in Bonn vergingen nur 5 Jahre, in denen Toeplitz angemessen arbeiten konnte. Zitieren wir daraus:

Es kam das Jahr 1933. Als „Altbeamter” - vor 1914 im Dienst - wurde er nicht gleich entlassen. Das geschah erst mit den „Nürnberger Gesetzen” des Jahres 1935. Er wurde pensioniert, nicht einmal emeritiert ... Täglich kamen erniedrigende Probleme, wie z.B. der Befehl, von nun an die Vorlesungen mit dem deutschen Gruß zu beginnen ... Allmählich rückten die Kollegen von ihm ab. Erst besuchte man sich nicht, dann grüßte man nicht mehr. Er konnte nicht mehr in die Universität, in die Bibliothek gehen ... Doch fand mein Vater auch eine, seine positive Antwort auf die Ereignisse und die wachsende Verfolgung. Er stellte seine Dienste der Bonner jüdischen Gemeinde zur Verfügung. Als Sachverständiger in Fragen der Erziehung übernahm er die Aufgabe, die jüdischen Kinder vor der nun feindlichen Umgebung zu bewahren und eine jüdische Schule zu gründen ... Für Jahre war er mit der Schule verbunden. Er wurde auch Hochschuldezernent der „Reichsvertretung der deutschen Juden”. Er unternahm es, begabte Studenten auszuwählen und auf Stipendien in Ausland zu schicken ... Er schrieb Artikel über jüdische Erziehung und reiste viel herum, besonders nach Berlin.

Es handelte sich um die private jüdische Volksschule in der Koblenzer Strasse 32, die 1935-1938 von Hans Herbert Hammerstein geleitet wurde [52, S. 41]. Erna Toeplitz beschreibt diese Schule ebenfalls in [5, S. 45]:

Schon im August überbrachte Rabbiner Levy meinem Mann die Bitte des Vorstandes, er möchte in einer Gemeinde-Versammlung eine Rede über die Gründung einer jüdischen Volksschule in Bonn halten, die von Samuel und Weiss ausging. Mit der ihm eigenen Intensität studierte mein Mann die Fragen der übrigen Schulen im Rheinland - nur in Bonn und Düsseldorf gab es keine - die finanziellen Fragen und das Lehrer-Angebot, und am 16.10.33 fand im Gemeindehaus die Gründungsversammlung der Schule statt. Sofort wurden 52 Kinder aus Bonn und benachbarten Gemeinden wie Beuel, Wesseling, Hersel etc. angemeldet.

Es gibt einen Brief von Toeplitz an Helmut Ulm vom 18. November 1935 [51, 4, der ebenso die Zeit 1933-1935 beschreibt:

Die Zeit war für mich - abgesehen von einer sehr häßlichen Episode - gar nicht so aufregend. Daß ich dieses Amt, wie es jetzt war, nicht mehr weiterzuführen brauche, ist für mich nahezu eine Befreiung. Obgleich mir nie was Unangenehmes zugestossen ist in diesen zwei Jahren, war diese ständige Bewußtheit, dieses Abhandenkommen jeder Naivität, eine Belastung, die mir immer erst dann ganz bewußt geworden ist, wenn ich davon einmal befreit war, und so auch jetzt. 
Im August 1935 schrieb Toeplitz an Courant [29, S. 79]:

Denn dies ist meine Auffassung: wir müssen die Stellen, auf denen man uns läßt, bis zum letzten Augenblick halten, nicht als ob eine Besserung in Sicht wäre - ausgeschlossen - sondern weil wir sonst in irgend einer Form der allgemeinen Judenheit zur Last fallen, mindestens einem anderen die Stellen wegnehmen. Ich betrachte es als ein Opfer, daß ich der Judenheit bringe, auf diesem Posten auszuhalten.

Otto Toeplitz war mit einigen Bonner Kollegen aus anderen Wissenschaften befreundet. Da war zum Beispiel der Geologe Hans Cloos und seine Familie. Cloos war 1926-1951 Direktor des Geologisch-Paläontologischen Instituts in Bonn. Zuvor hatte er in Marburg und Breslau gearbeitet. Cloos war ein dezidierter Gegner der Nationalsozialisten. Er hatte 1933 auf einer Reise nach Afrika den Sohn Walter Toeplitz besucht, der 1933 nach Südafrika ausgewandert war. Am Tag vor der Reichskristallnacht brachte er Otto Toeplitz nach Aachen in Sicherheit, damit er dem Konzentrationslager entgehen konnte. Nach dem Krieg wurde Cloos von den Besatzungsmächten für den Wiederaufbau gebraucht und sogar als Bürgermeister von Bonn ins Spiel gebracht [52, S. 133]. Paul Ernst Kahle, ein Bonner Orientalist, und seine Frau Marie haben ebenfalls eine besondere Rolle als Helfer in der Zeit der Verfolgung gespielt, und beide haben dies in Berichten dokumentiert 13 .

Ein Artikel mit dem Titel „Was macht der jüdische Abiturient ?” erschien in der Zeitung des Central-Vereins [43. Darin gibt Toeplitz Ratschläge zur Berufswahl von jüdischen Abiturienten. Dieser Artikel vom März 1937 strahlt noch einen gewissen Optimismus aus, wie Toeplitz selbst im letzten Satz betont, jedoch deutlich und vielsagend als ,eingeschränkten Optimismus“ benennt. Er konzentrierte sich beim Inhalt auf Jungen, wie die folgende kuriose Passage mit Kommentar der Redaktion unterstreicht:

Mädchen sollen unter den heutigen Umständen jedes Studium, dessen Ziel eine produktive wissenschaftliche Betätigung ist, unterlassen. (Wir können der Meinung des Verfassers in der von ihm gewählten apodiktischen Form nicht ganz beipflichten. Hervorragend begabte Mädchen sollten auch heute den Wettbewerb mit männlichen Bewerbern aufnehmen (Die Schriftleitung).)

Otto Toeplitz hat in dieser Zeit, wie man sieht, nicht aufgegeben und vermutlich auch bis 1937 mathematisch weiter gearbeitet, wie Köthe in [4, S. 22] berichtet. Im Sommer 1938 besuchte er Palästina auf Einladung von Uri und trug dort unter anderem an der Jerusalemer Universität vor. Von Seiten der Administration wurde ihm eine Stelle in Aussicht gestellt [51, S. 113]. In dieser Zeit muss wohl die Entscheidung gefallen sein, endgültig nach Palästina auszuwandern. Andere Reisepläne in die USA werden von Richard Courant [21, S. 213-214] und etwas ausführlicher von Uri Toeplitz [51, S. 119] geschildert, Es scheint, dass die Auswanderung nach Jerusalem offenbar auch unsicher war. Nach der Rückkehr nach Deutschland verschärfte sich der Antisemitismus in Deutschland noch mehr. Die Hilfe von Hans Cloos während der Reichskristallnacht war womöglich lebensrettend. Uri Toeplitz berichtet in [4, S. 30] von einer Vorladung durch die Gestapo im Winter 1938/39, die die Gesundheit des Vaters weiter beschädigte.

In einem Brief an Heinrich Behnke vom 1. Januar 1939 beschreibt Toeplitz die Zeit des Wartens vor der Abreise nach Jerusalem [44: 
Meine Sache liegt z.Z. in den Händen der engl. Regierung zu Jerusalem. Es sind bange Wochen des Wartens, bange auch deshalb, weil ich indessen meine hiesigen Bücher abbrechen muss, um nach Eintreffen des Zertifikats nicht zuviel Zeit mit den $\ddot{A}$ tern zu verlieren. Die Ämter sind bisher alle sachlich und höflich.

In diesem Brief kündigte er auch einen Abschiedsbesuch in Münster an, der tatsächlich Anfang Februar stattfand, wie Gottfried Köthe in [4, S. 23] eindringlich beschreibt:

Wenige Tage vor seiner Ausreise kam Toeplitz noch nach Münster, wo Behnke und ich ihn am Bahnhof abholten und mit ihm in ein naheliegendes Café gingen. Toeplitz war gekommen, um mit uns, seinen Freunden, Abschied zu nehmen. Ich sehe ihn noch vor mit, aus dem abfahrenden Zug uns zuwinkend, ernst und traurig. Wir ahnten, daß es ein Abschied für immer war.

Erna und Otto Toeplitz verließen Deutschland im Februar 1939, getrennt über die Schweiz [52, S. 74-75]. Es existieren einige Briefe aus dem Jahr 1939, die nochmals die menschlichen Qualitäten von Otto Toeplitz beleuchten. So gibt es zwei Briefe von Polya aus dem Mai 1939, in denen sich beide über Schur und Hausdorff austauschen. Es ist bekannt, dass Toeplitz genau Buch führte (genauer gesagt Karteikarten, deren Verbleib mir nicht bekannt ist) über die Schicksale und Selbstmorde von jüdischen Kollegen. In einem Brief an Max Born schreibt er 1939 552, S. 84]:

Am 1.2.39 habe ich in Basel die Grenze überschritten und bin seit dem 10.2.39 in der Verwaltung der hiesigen Universität als wissenschaftlicher Berater tätig ... Von diesem Hexenkessel, Deutschland, will ich nicht viel schreiben. Derjenige, der 1933 mitgemacht hat, wie Du, hat noch lange keine Vorstellung von 1938/1939. Worte können nicht wiedergeben, in welche Verzerrung dieses vortreffliche Volk geraten ist.

Im Rest des Briefes spricht Toeplitz über seine neue Tätigkeit, die nicht leicht sei, trotz der Unterstützung durch den Förderer Salman Schocken, den Chef der Verwaltung der Hebräischen Universität zu Jerusalem. Nach einem Jahr in Jerusalem starb Otto Toeplitz am 15. Februar 1940 mit 58 Jahren in Jerusalem, nachdem seine Gesundheit sich wieder verschlechtert hatte. Er wurde auf dem Ölberg beerdigt. Zu seinen Ehren hängt seit 1995 [5] eine Gedenktafel im Mathematischen Institut der Universität Bonn.

\section{Der Algebraiker Hilbert}

Otto Toeplitz schrieb 1922 einen Artikel über David Hilbert mit dem Titel „Der Algebraiker Hilbert" 40. Aus dem ganzen Artikel spricht die Bewunderung von Toeplitz für Hilbert. Nachdem er zunächst die Arbeiten Hilberts zur Zahlentheorie als den Kern von Hilberts Leistungen würdigt, geht er auf dessen Leistungen in der Algebra ein:

Von dem Algebraiker Hilbert zu erzählen, so zu erzählen, dass der Naturwissenschaftler ein Bild erhält, und selbst derjenige einen Eindruck, der dessen mathematische Exerzitien bei der Gleichung der Ellipse aufgehört haben, ist vermessen ... Indessen Hilbert nimmt zur Algebra eine besondere Stellung ein. Seine Methoden sind von einer ganz anderen Art wie die eines Kronecker, Frobenius, Gordan; es sind Methoden, auf die bei ihrem Auftreten keiner der Beteiligten gefasst war ... Und dieser Schimmer einer Möglichkeit, von dem Algebraiker Hilbert zu erzählen, 
soll deshalb nicht ungenutzt bleiben, weil sich in seinen algebraischen Forschungen ein Wesenszug offenbart, der sich von dem Hintergrunde der Algebra plastischer abhebt als von dem seiner vielen Arbeitsgebiete, und der ein Grundpfeiler ist für die Analyse des mathematischen Menschen Hilbert.

In der Folge geht Toeplitz ausführlich auf die Leistungen Hilberts in der Invariantentheorie ein. Im letzten Teil des Artikels schlägt er allerdings die Brücke zum Gebiet der Integralgleichungen und der unendlichen Matrizen, Toeplitz eigenem Arbeitsgebiet:

Aber Hilbert hat noch einmal eine umfassende algebraische Leistung vollbracht, in der Theorie der Integralgleichungen und unendlichvielen Variablen, deren analytische Seite an anderer Stelle dieses Heftes zur Geltung kommt ... Das einigende Band für alle diesen Theorien ist ein algebraischer Gedanke ... So hat Hilbert in seiner Theorie der unendlichvielen Variablen ein großes Haus errichtet, in dem viele einzelne Untersuchungen geräumige Wohnungen gefunden haben; ... So steht der Algebraiker Hilbert in ausgeprägter Eigenart vor uns, wie nicht ein jeder Mathematiker.

Aus dieser Beschreibung liest man die Bewunderung für Hilbert heraus, es wird aber auch deutlich, wie sehr Toeplitz seine eigene Forschung vor einem algebraischen Hintergrund sah. Im letzten Abschnitt des Artikels verbindet Toeplitz die beschriebenen Wesenszüge von Hilbert mit seiner Exaktheit und dessen Beurteilung der Mathematik in den berühmten Pariser Problemen und der Grundlagenfrage der Mathematik. An dieser Stelle verteidigt Toeplitz den formalen axiomatischen Standpunkt Hilbert gegenüber Weyl, Brouwer und anderen, die dem Konstruktivismus nahestanden [22]. Dies ist schon eine Vorwegnahme von Teilaspekten des späteren Annalenstreits.

\section{Briefe von Klein und Hilbert: JÜDische Berufungslisten}

Jüdische Mathematikprofessoren in Deutschland spielten erst seit dem 19. Jahrhundert eine Rolle, angefangen mit Moritz Abraham Stern, einem Schüler von Gauss und dem ersten jüdischen Ordinarius. Im Fall von Carl G.J. Jacobi hat Felix Klein bemerkenswerte Aussagen in diesem Zusammenhang gemacht. Nachdem er zuvor viele kleine Spitzen über die Person von Jacobi in den Text einstreut, und die wissenschaftlichen Leistungen und die Schule Jacobis sehr hoch bewertet, schreibt er [14, S. 114]:

Ehe ich die Betrachtungen über Jacobi schließe, möchte ich noch eine Tatsache erwähnen, die mir unter dem Gesichtspunkt der Charakteristik dieses Mannes als auch unter dem der Entwicklung unserer Wissenschaft nicht unwichtig erscheint. Bekanntlich hatte das Jahr 1812 die Emanzipation der Juden in Preußen gebracht. Jacobi ist der erste jüdische Mathematiker, der in Deutschland eine führende Stellung einnimmt. Auch hiermit steht er an der Spitze einer großen, für unsere Wissenschaft bedeutungsvollen Entwicklung. Es ist mit dieser Maßnahme eine neues, großes Reservoir mathematischer Begabung für unser Land eröffnet, dessen Kräfte neben dem durch das französische Emigrantentum gewonnenen Zuschuß sich in unserer Wissenschaft sehr bald fruchtbar erweisen. Es scheint mir durch solch eine Art Blutserneuerung eine starke Belebung der Wissenschaft gewonnen zu werden.

David Rowe thematisiert in 25 die Berufungsangelegenheiten um die Jahrhundertwende in das zwanzigste Jahrhundert, und damit zusammenhängend die „Jewish 
Question", besonders anhand der Person Adolf Hurwitz. Hurwitz Ruf nach Göttingen und die Rollen von Felix Klein, David Hilbert und anderen in dieser Zeit werden beleuchtet. Für Toeplitz waren Hilbert und Klein nicht nur mathematische Vorbilder, er hat sie auch in seinen eigenen Berufungsaktivitäten als Gutachter bemüht. Man kann sich denken, dass Klein und Hilbert bei vielen Berufungen in der Mathematik entscheidenden Einfluss nehmen konnten. David Hilbert schrieb an Toeplitz am 8. Februar 1920 einen Brief, in dem er auf ein Berufungsverfahren in Kiel auf eine Professur für Algebra eingeht [44:

An erster Stelle würde ich Steinitz vorschlagen, der es verdient und der kommt und mit dem sie ausgezeichnet fahren werden (Fussnote: den ich auch rein wissenschaftlich für den erfolgreichsten Forscher unter den Genannten halte.). Wenn Sie einen nicht-jüdischen Mathematiker an 2ter oder 3ter Stelle nennen wollen, so kommen Bieberbach, für den ich übrigens keineswegs schwärme, und Hausdorff (Anmerkung: sic!) in Frage ... Hellinger ist ein Faulpelz und soll erst wieder etwas machen, ehe man ihn auf die Liste setzt, ebenso Dehn. Übrigens werden alle in nächster Zeit Rufe bekommen, da ein großer Mangel an Mathematikern besteht.

Steinitz hat die Stelle auch bekommen. Sein mathematischer Ruf begründete sich auf seine Arbeiten zur Theorie der algebraischen Körpererweiterungen [31. Er hatte später auch anerkannte Leistungen in der Polyedertheorie erbracht und starb 1928 in Kiel. Erstaunlicherweise hatten Nachkommen der Familien Steinitz und Toeplitz in Jerusalem Kontakt, wie man aus Dokumenten im Internet (http://family.steinitz.net) entnehmen kann. Felix Klein hat in einem bemerkenswerten Brief an Toeplitz am selben Tag, dem 8. Februar 1920, auch zur selben Sache Stellung genommen. Wir geben nur das Ende des Briefes wieder, weil es die Art und Weise deutlich macht, mit der Klein agierte [4]:

Nun komme ich, um nichts zurückzuhalten, auf die Frage des Antisemitismus. Sie wissen, wie ich es selbst immer gehalten habe, seit ich 1874 die Berufung von Gordan nach Erlangen veranlaßte: mir war der einzelne Jude willkommen, indem ich voraussetzte, daß er mit den übrigen Mitgliedern der Universität kooperieren werde. Aber nun haben sich im Laufe der Zeit die Gegensätze prinzipiell verschärft. Wir haben auf der einen Seite nicht nur ein ungeheures, der merkwürdigen Leistungsfähigkeit entprechendes Vordrängen des Judentums, sondern das Hervorkommen der jüdischen Solidarität (welche dem Stammesgenossen auf alle Weise in erster Linie zu helfen strebt). Dazu nun als Rückwirkung den starken Antisemitismus. Das Problem ist ein allgemeines, bei dem Deutschland, soweit nicht gerade die moderne östliche Einwanderung in Betracht kommt, nur eine sekundäre Rolle spielt. Niemand kann sagen, wie sich das Ding weiterentwickelt. Aber ich mache darauf aufmerksam, daß die sämtlichen fünf Gelehrten, die Sie für Ihr Ordinariat in Aussicht nehmen, jüdischen Ursprungs sind. Ist das eine zweckmäßige Politik ? Ich nehme von vorneherein an, daß Sie das nicht beabsichtigt haben. Man kann auch beinahe so argumentieren: daß der an allen Universitäten etc. latent vorhandene Antisemitismus die christlichen Kandidaten so bevorzugt habe, daß nur noch jüdische zur Verfügung stehen. Aber ich bitte doch darüber nachzudenken. Wir treiben möglicherweise in Gegensätze hinein, die für unsere gesamten Zustände unheilvoll werden könnten.

In unserer heutigen Zeit wirken manche dieser Worte, wohl als Abbild des „Zeitgeistes”, klarerweise „politisch inkorrekt”. Klein spricht den überall vorhandenen Antisemitismus jedoch klar aus. Erstaunlich ist das Vorhersehen von Entwicklungen, besonders im letzten Satz. Uri Toeplitz geht auf diesen Brief sowie weitere 
beteiligte Personen wie Eduard Study und Edmund Landau ebenfalls in 51, S. 139-141] ein.

\section{Heinrich Behnke und die Semesterberichte}

Heinrich Behnke, der in Münster seine ganze Wirkung und Bedeutung entfaltete, kam aus Hamburg von Erich Hecke, wo auch Toeplitz regelmäßig zu Gast war. Behnke schreibt in [2, S. 48/49], dass Hecke manchmal leicht abfällig vom „dicken Toeplitz" sprach. Behnke erwähnt darin auch, dass Toeplitz Sinn für den Unterricht ausgeprägter war, als das im Kreis der Hilbertschüler üblich war. Erich Hecke war Schüler von David Hilbert. Besonders interessant in Behnkes Autobiographie [2] sind die Beschreibungen des Universitätslebens am Anfang des 20ten Jahrhunderts. Trotz des Titels „Semesterberichte” enthält sie ansonsten leider nur wenige Erinnerungen an Toeplitz. Nachdem Toeplitz in Kiel bereits Fortbildungsveranstaltungen für Mathematiklehrer durchgeführt hatte 41, seine beachteten Vorträge über die Schnittstelle Schule-Universität [37, 38] gehalten hatte und auch häufig als Gast der 1919 gegründeten Hamburger Universität mit Behnke in Kontakt war, gab es in der Bonner Zeit ab 1927 regelmäßige Treffen mit Behnke und seinen Schülern. Gottfried Köthe beschreibt ein Zusammentreffen von Behnke und Toeplitz 1929 in Bonn in [4, S. 10]:

Als Toeplitz bei Emmy Noether anfragte, ob sie ihm einen jungen Algebraiker empfehlen könnte, schlug sie mich vor, und ich kam im Sommer-Semester 1929 zu einem Vorstellungsbesuch nach Bonn. Ich geriet in ein großes Treffen von Toeplitz und Behnke mit ihren Schülern und machte gleich eine Rheinfahrt mit.

Im Jahr 1932 gründeten Behnke und Toeplitz die Zeitschrift „Semesterberichte zur Pflege des Zusammenhangs von Universität und Schule". Im Universitätsarchiv Münster befindet sich ein Geleitwort zum Erscheinen der Semesterberichte vom Juni 1932 [11, S. 201]:

Ich habe es immer als schmerzlich empfunden, dass seit der Aufgabe der Ferienkurse faktisch keine Beziehung mehr zwischen den Studienräten der Mathematik und uns besteht. Das ist für den mathematischen Unterricht an Schulen und Universität gleich schädlich. Da andererseits infolge der weiteren Beschränkung der Mittel die Wiederaufnahme der Ferienkurse nicht möglich ist, haben Herr Kollege Toeplitz aus Bonn - der diesen Zustand ebenso stark empfindet - und ich beschlossen, Semesterberichte herauszugeben.

Die ersten Hefte der Semesterberichte begleiteten die von Behnke und Toeplitz organisierten ,Tagungen zur Pflege des Zusammenhangs von Universität und höherer Schule", die ab 1931 in Münster stattfanden [10, S. 34]. Heinrich Behnke beschreibt die Gründung der Semesterberichte auch im Nachhinein in [3, S. 6]:

1932 wurden mit dem Verfasser dieser Zeilen trotz der großen Wirtschaftskrise die alten Semesterberichte in ihrer bescheidenen Form der blauen hektographierten Hefte (1-14) begründet. Der äußere Anlaß dafür war unser Verlangen, mit unseren früheren Studenten auch noch in Verbindung bleiben zu können, wenn sie zur Schule zurückgekehrt waren. Auf den Gedanken, daß ein erheblicher Teil unserer uns enger verbundenen Studenten in der Industrie Beschäftigung suchen sollte oder an die Hochschulen des In- und Auslandes gehen würde, kam damals noch keiner von uns. Das Programm der Blätter war im wesentlichen das der heutigen Semesterberichte (die seit 1949 bei Vandenhoeck und Ruprecht bzw. im Springer Verlag 
erscheinen). Doch wer die Reihe der 14 Hefte verfolgt, die bis zum Kriege veröffentlicht wurden, erkennt, daß Toeplitz noch eine weitere Linie verfolgen wollte, als die Semesterberichte dann wirklich eingeschlagen haben. Er hatte die Absicht, in diesen Blättern sich auch über die Fragen des Schulunterrichts auszulassen. Doch beklagte er dann, daß offenbar Fachgenossen und Schulleute wenig darauf eingingen.

Diese Textpassage bestätigt die gleichberechtigte Rolle von Toeplitz bei den Semesterberichten. Toeplitz wurde ab 1935 aus der Redaktion durch Einfluss von Georg Hamel und Theodor Vahlen entfernt [10, S. 193]. Im Jahr 1939 wurde die Zeitschrift eingestellt. Die Gründe dafür waren zum einen finanzieller Art, aber es gibt auch Stimmen, die davon ausgehen, dass Ludwig Bieberbach die Reduktion der Fachzeitschriften in der DMV so vorangetrieben hatte, dass die Semesterberichte politisch unter Druck gerieten [10, S. 209], [24, S. 19]. Erst nach dem Krieg wurden die Semesterberichte 1949 unter dem neuen Namen „Mathematisch-Physikalische Semesterberichte" bei Vandenhoeck und Ruprecht wieder von Behnke zusammen mit Wilhelm Süß und Walter Lietzmann herausgegeben. Sie existieren bis zum heutigen Tage, derzeit unter dem Namen „Mathematische Semesterberichte” beim Springer Verlag.

Über das persönliche Verhältnis zwischen Behnke und Toeplitz wissen wir nicht sehr viel, ebensowenig, ob es sich nach 1933 verändert hat. Die erste Frau von Heinrich Behnke, Aenne Albersheim, war jüdischer Abstammung und verstarb 1927 nach Geburt des Sohnes Hans. Insofern war Behnke sicherlich beim Thema Antisemitismus sensibel. Uri Toeplitz geht darauf in [51, S. 133] ein:

Behnke war ein häufiger Gast bei uns in Bonn, immer gute Laune mit sich bringend, wie dies auch mit seiner imposanten Figur und Stentorstimme zusammenging. Er kam weiter zur Nazi-Zeit, obwohl er, der aus erster Ehe einen halb-jüdischen Sohn hatte, wohl besonders vorsichtig sein musste.

In Behnkes Autobiographie findet sich davon wenig. Es ist hervorzuheben, dass Behnke bis zu Toeplitz Abreise nach Jerusalem, ebenso wie Gottfried Köthe, mit Toeplitz persönlichen und brieflichen Kontakt hatte. Behnke hat offenbar ab 1933 dennoch eine im Grunde ängstliche politische Einstellung gehabt. Dies wird durch Peter Thullen in seinen Tagebüchern hervorgehoben [33, S. 48], siehe auch [10, S. 42]. Volker Remmert erwähnt in [24, S. 12] Aktennotizen des Münsteraner Rektorats, die belegen, dass Behnke zwar unter Beobachtung stand, aber aufgrund seiner Verdienste einen Schutz genoss. Es gibt einen Hinweis auf Verstimmungen zwischen Behnke und Toeplitz, den Uta Hartmann in Ihrer Dissertation [10, S. 37] aufgebracht hat. In einem Brief an Karl Jaspers vom 19. Oktober 1936 schreibt Toeplitz über einen Vorfall, der jedoch im Unklaren bleibt:

Es handelte sich darum, Behnke in einem kritischen Augenblick zu erziehen, wo er es wieder mal nötig hatte. Schwer genug in meiner Lage, aber ich wollte es schon. Hätte er an Ihnen wie an allen anderen keine Stütze gefunden, so hätte ich sicher mehr erreicht. Ich sehe eine Menge Arier in ähnlicher Lage, und B.[ehnke] mit seiner ungemeinen und biegsamen Intelligenz müsste es schaffen, ebenso klar dazustehen, wie manche von diesen, die ich im Auge habe. Aber es rächen sich jetzt bei ihm einige große Fehler, gegen die ich immer angekämpft habe ... Mir ist eine solche Beziehung von geringer Innerlichkeit eigentlich trauriger, als gar keine. 
Es ist die Frage, und bleibt offen, wie tief diese Verstimmung bei Toeplitz war, zumal die beiden Persönlichkeiten sehr unterschiedlich waren. Umgekehrt finden in den noch existierenden Briefen von Behnke an Jaspers solche Stimmungslagen keine Erwähnung [10, S. 38].

\section{Toeplitz und DiE MAThematikgeschichte}

Bereits in Kiel hatte Toeplitz intensive Kontakte zu Heinrich Scholz und Julius Stenzel und veranstaltete mit ihnen gemeinsam ein mathematisch-historisches Seminar, in dem vor allem antike Mathematik besprochen wurde. Stenzel war ein angesehener Graezist, Scholz ein Theologe und Philosoph, der sich später zum Logiker wandelte und eine Schule in Münster begründete. In den Bonner Jahren baute Toeplitz zusammen mit Erich Bessel-Hagen eine mathematik-historische Bibliothek auf [5, S. 30] und hielt auch dort ein historisches Seminar ab. Zu diesem Bonner Kreis um Toeplitz gehörte später auch Oskar Becker, der 1931 nach Bonn berufen wurde, seine Eudoxosstudien dort entwickelte und in den Quellen und Studien [20] publizierte. In den Semesterberichten hat Harald Boehme über Oskar Becker kürzlich einen Artikel verfasst [6]. Die Staatsexamensarbeit von Daniel Schneider [26] behandelt ebenfalls verschiedene Aspekte dieser Person. Oskar Becker entwickelte in den dreißiger Jahren eine deutliche nationalsozialistische Gesinnung, die dafür sorgte, dass er nach dem Krieg von den Besatzungsmächten für einige Jahre in den einstweiligen Ruhestand versetzt wurde. Wie diese Ideologie mit seiner vorangegangenen Zusammenarbeit mit Toeplitz vereinbar ist, bleibt uns heute unverständlich.

Es ist erstaunlich, dass an einigen Instituten in dieser Zeit Mathematiker gleichzeitig intensive historische Interessen entwickelten und Seminare dazu abhielten. Der Krieg hat diese Entwicklung aber wieder beendet. Es wäre möglicherweise interessant, die Ergebnisse dieser Seminare noch mehr aufzubereiten. Bei Toeplitz wurde oft darauf hingewiesen, beispielsweise von Max Born, dass seine Breslauer familiären Wurzeln und seine humanistische Schulbildung die Grundlage für diese Neigung bildeten. Jedoch war sein Interesse in dieser Hinsicht weit überdurchschnittlich. Der bemerkenswerte Kontakt zu den Philologen wurde von Toeplitz selbst als Arbeitsprogramm thematisiert [46, S. 4]:

Die Forschung ist hier vor den Toren des Baues, als den wir die griechische Mathematik vorstellen wollen, stehen geblieben. Nur ein neues System der Zusammenarbeit von Philologe und Mathematiker kann diese Tore öffnen.

Toeplitz hatte also nicht nur ein ausgeprägtes Interesse an mathematisch-historischen Fragen, sondern auch ein Bedürfnis, mit adäquaten fächerübergreifenden Methoden zu arbeiten. Julius Stenzel wurde 1925 nach Kiel berufen. Er hatte mit seinem Buch 32 zu Plato und Aristoteles die Arbeiten von Toeplitz [45, 46, 47] zur griechischen Mathematik beeinflusst. In diesen Artikeln begab sich Toeplitz intensiv in den Diskurs, der zu diesen Themen in seiner Zeit herrschte, zum Beispiel durch Oswald Spenglers „Der Untergang des Abendlandes”, und bewies oder widerrief Thesen, indem er genaue Übersetzungen anforderte, um den Interpretionsspielraum kritisch zu kontrollieren. So sollten zum Beispiel Fehlinterpretationen durch Analogien mit moderneren mathematischen Begriffen ausgeschlossen werden. Durch diese Vorgehensweise kam er zu einer Kritik an Thesen von A. E. Taylor. In eine ähnliche Auseinandersetzung begab sich Toeplitz mit seiner Widerlegung der Kritik von Ernst Mach an der Darstellung des Archimedischen Beweises des Hebelgesetzes [17. Zwei seiner Doktoranden, Walter Stein [30] und Dora Reimann 23], publizierten über Toeplitz Kritik an Mach in den Quellen und Studien, was einer massiven Kritik an 
Mach gleichkam. In der Staatsexamensarbeit von Sebastian Lange [16] wird dies ausführlich behandelt.

Die Breite von Toeplitz Denken wird auch offenbar durch die engen Kontakte, die Toeplitz in Bonn mit Professorenkollegen aus anderen Gebieten pflegte: da war Hans Cloos (Geologe), Richard Thoma (Jurist), Paul Ernst Kahle (Orientalist), Leo Waibel (Geograph), Fritz Kern (Historiker) und Adolf Zycha (Rechtshistoriker) [5. S. 28]. In letzter Konsequenz hatte Toeplitz zusammen mit Julius Stenzel und Otto Neugebauer 1929 die Zeitschrift „Quellen und Studien zur Mathematik, Astronomie und Physik" gegründet, die von 1931 bis 1938 beim Springer Verlag erschien. Hier ein Zitat aus dem Geleitwort zur ersten Ausgabe, das wieder die übergreifenden Ideen von Toeplitz in Bezug auf Historie und Didaktik beleuchtet [20, Band 1, S. 1]:

Die heute so aktuellen Bemühungen um die Grundlagen der Mathematik, das damit eng zusammenhängende Interesse an philosophischen und didaktischen Problemen haben mit gutem Recht auch die Frage nach dem geschichtlichen Werdegang mehr in den Vordergrund geschoben. Wir glauben daher, den Versuch wagen zu dürfen, der Forschungen nach den geschichtlichen Grundlagen der mathematischen Wissenschaften eine neue Stätte zu bieten.

In den Quellen und Studien erscheinen Artikel von Erich Bessel-Hagen, Oskar Becker und Bartel Leendert van der Waarden. Die Zeitschrift ist die zweite, die Toeplitz gründete, und bei der er seinen hohen Anspruch an die Mathematikgeschichte und Philologie einflussreich etablierte. Der Mitherausgeber Otto Neugebauer emigrierte 1933 nach Kopenhagen und Julius Stenzel starb 1935 in Halle. Die Zeitschrift lief daher aus, da Toeplitz ohnehin als jüdischer Herausgeber Ablehnung erfuhr. Oskar Becker machte sich im Dezember 1938 in einem Brief an Otto Neugebauer sogar für die Absetzung von Toeplitz als Herausgeber stark, worauf Neugebauer aus Kopenhagen eine scharfe Antwort schrieb [29, S. 142] und seinen eigenen Rücktritt ankündigte:

Sie schrieben mir, daß Sie als Nationalsozialist anscheinend eine von der meinen verschiedene Auffassung haben, trotz ihrer persönlichen Hochachtung vor Herrn Toeplitz. Ich kann darauf nur antworten, daß ich nicht im glücklichen Besitz irgendeiner "Weltanschauung" bin und daher genötigt bin, mir in jedem einzelnen Fall zu überlegen, was ich tun soll, ohne mich auf ein von vorneherein gegebenes Dogma zurückziehen zu können. Diesen Nachteil dem praktischen Leben gegenüber wiegt vielleicht auf, daß es mir erspart bleibt, mich von den Menschen, die ich hochschätze, deshalb trennen zu müssen, weil sie das Unglück haben, von anderen Menschen mißhandelt zu werden.

In diesen Zeilen eines bewundernswerten Charakters steckt nicht nur die Verteidigung von Otto Toeplitz, sondern auch die kaum versteckte Verachtung der Gedankenwelt dieses Oskar Becker, der durch seine Forderung vom Mitläufer zum Täter wurde.

\section{Die Genetische Methode}

In einem Vortrag am 24. September 1926 vor dem Reichsverband [37] legte Otto Toeplitz in großer Klarheit die hochschuldidaktischen Probleme in der Anfängervorlesung „Infinitesimalrechnung” dar. Er berief sich dabei auf eine ähnliche Auseinandersetzung, die auf der Tagung der Naturforscher und Ärzte 28 Jahre früher im Zusammenhang mit Vorträgen von Alfred Pringsheim und Felix Klein stattfand 
[37, S. 88]:

Der tatsächliche Zustand dieser Vorlesung an den deutschen Universitäten zeigt noch heute die gleiche bunte Mannigfaltigkeit; auf der einen Seite die strenge Observanz, die mit einer sechswöchentlichen Dedekindkur anhebt und dann aus den Eigenschaften des allgemeinen Zahl- und Funktionsbegriffs die konkreten Regeln des Differenzierens und Integrierens herleitet, als wären sie notwendige, natürliche Konsequenzen, auf der anderen Seite die anschauliche Richtung, die den Zauber der Differentiale walten läßt und auch in der letzten Stunde der zwei Semester umspannenden Vorlesung den Nebel, der aus den Indivisibilien aufsteigt, nicht durch den Sonnenschein eines klaren Grenzbegriffs zerreißt; und dazwischen die hundert Schattierungen von Diagonalen, die man zwischen zwei zueinander senkrechten Ideenrichtungen einzuschalten vermag.

Toeplitz versucht in [37, dieses Problem zu erklären. Er führt dazu drei sogenannte Momente an: Das erste Moment ist die Kluft zwischen Exaktheit und Anschaulichkeit, die besonders in der Mathematik steckt, seit der Anspruch der Exaktheit dort so wichtig geworden ist. Toeplitz führt Weierstraß als Vorreiter der mathematischen Strenge an. Das zweite Moment ist in der Rolle der Anfängervorlesung zwischen Schule und weiterführenden Kursvorlesungen begründet. Dies zeigt sich in der weiten Spanne zwischen praktischen Rechen- und Beweistechniken und z.B. der Epsilontik, d.h. dem strengen Grenzwertbegriff. Dieses inhaltliche Problem wird überlagert von der Inhomogenität an Begabung der Hörer einer solchen Vorlesung. Er trifft die Entscheidung, dass die $45 \%$ der mittelguten Hörer (ohne die $5 \%$ der absolut Begabten und die $50 \%$ der Unbegabten) im Mittelpunkt des Interesses stehen müssen. Das dritte Moment ist die Überschneidung mit der Schule, heute Schnittstelle genannt. Die Art und Weise wie in der Schule Differentialrechnung gelehrt werde, sei auf das Gros der Schüler abgestellt, so dass die Schule das Problem nicht unbedingt verbessere. Toeplitz schlägt in der Folge eine Lösung dieser Probleme dar, die sich die ,genetische Methode” nennt. Diese Methode stellt auf eine motivierende Herangehensweise an Begriffe ab, ähnlich der historischen Entstehungsgeschichte. Er differenziert aber klar von einer „historischen Methode”, so dass es hier nicht darum geht, Begriffe in ihrer historischen Entwicklung zu beschreiben, sondern das Herangehen in der Entstehung (Genese) zu verbessern. Er sieht das nicht als neue Idee, sondern erinnert wieder an die Diskussion zwischen Klein und Pringsheim, wo auf das „biogenetische Grundgesetz” hingewiesen wurde. Es folgt ein Beispiel zur genetischen Methode an Hand der Reihenkonvergenz, bei dem historische Beispiele von Reihen (Mercator, Newton, Euler, Bernoullis) einer Konvergenzbetrachtung vorangestellt werden. Otto Toeplitz hat in Vorlesungen und mit Hilfe seiner Arbeitsgruppe an einem tatsächlichen Umsetzungskonzept gearbeitet, das posthum von Gottfried Köthe publiziert wurde [34. Die Folgen aus den drei Momenten werden schließlich in drei Thesen zusammengefasst: Zum ersten sei die Integralrechnung vor der Differentialrechnung zu behandeln, was durch die Griechen (Archimedes) und Dedekind, Fermat und Cavalieri untermauert wird. Die zweiten These stellt den Hauptsatz der Differential- und Integralrechnung in den Mittelpunkt. Anhand der Personen Fermat, Barrow, Newton und Leibniz wird der Unterschied zwischen Genesis und Historie für Toeplitz nochmal deutlich, und man spürt seine Gelehrtheit, wenn er den Prioritätsstreit in die historische Ecke stellt, und die Genesis betont. Seine dritte These ist die Behauptung, dass Grundbegriffe (wie der Zahlbegriff) in das zweite Semester gehören. Als Zusatz bemerkt er, dass der Cantorsche Zahlbegriff dem von Dedekind vorzuziehen sei, nachdem er eine Fundamentalkritik an den Dedekindschen Schnitten vorgenommen hat. Wenngleich die 
Idee der Schnitte im Wesentlichen schon in der griechischen Mathematik auftaucht, wie Toeplitz in [37, S. 97] anmerkt, lehnt er diese in der Anfängervorlesung aus wohlbekannten didaktischen Gründen ab. Die Schönheit der Idee von Dedekind, und die Möglichkeit eines genetischen Zugangs zu ihr ignoriert er, weil er Dedekind Ungenauigkeiten unterstellte, die aus heutiger Sicht behebbar sind. Als vierte These will Toeplitz die Differentialrechnung nur noch bei „rechnerischen” Funktionen im Gegensatz zu ,willkürlichen” Funktionen sehen. Dies mündet in einen historischen Exkurs in die Welt von Barrow, Leibniz und Newton, mit der These, dass die Neuerungen von Leibniz und Newton sich nur auf die Weiterentwicklung zum Kalkül, also die Berechenbarkeit beziehen. Diese These zeigt wieder die historischen Interessen von Toeplitz. In dem eigentlichen Buch [34 wird zu Anfang eine schrittweise Einführung in die Gedankenwelt gegeben. Erst in $§ 9$ wird der Grenzwertbegriff exakt eingeführt und viele der üblichen Beweise aus der Epsilontik behandelt. Auf diesem Grenzwertbegriff für Folgen baut dann der Begriff der Reihe und des Integral auf. Das Ende des Buches bringt eine Diskussion der Keplerschen Gesetze. Überhaupt enthält der Stoff viele physikalische Anwendungen, Übungsaufgaben, die Toeplitz so wichtig waren, sowie viele historische Bemerkungen und motivierende Abschnitte. Das bemerkenswerte Buch ist womöglich vielen Analysisbüchern der heutigen Zeit überlegen. Die genetische Methode hat sich seitdem verselbstständigt und wird in vielen Kontexten, neben der historisch-genetischen auch in einer psychologischgenetischen Variante erwähnt 27. Toeplitz Zugang bezieht sich jedoch eindeutig auf die historisch-genetische Richtung, wenngleich er auch die Abgrenzung zum rein historischen Zugang wünschte. Der genetische Zugang von Toeplitz hat natürlich auch seine Nachteile. Heinrich Behnke macht dazu in [3, S. 4] eine Bemerkung:

Die einzelnen Fundamentalsätze sollen historisch entwickelt und dadurch dem Ḧ̈rer in einer natürlichen Spannung und zugleich pädagogisch naheliegenden Weise vorgetragen werden. Es liegt nahe zu fragen, wie dazu die erforderliche Zeit gewonnen werden kann.

Der Schluss des Artikels von Toeplitz wendet sich gegen die Suche nach der absoluten Objektivierung in der Mathematik. Toeplitz reiht Hilbert und Weyl in sein Denkschema ein. Man merkt bereits in diesem Aufsatz, wie sich Toeplitz von seinen Vorbildern Klein und Hilbert abzugrenzen versucht.

\section{Die Schnittstelle ZWISChen Schule Und Universität}

Das Problem der Schulmathematik behandelte Toeplitz bereits in seiner Rede 37] von 1926. Zwei Jahre später hielt er einen Vortrag auf der Tagung der Naturforscher und Ärzte [38, der sich genauer mit der Schnittstelle Schule-Hochschule beschäftigte. Als Hintergrund muss man wissen, dass sich das Schulcurriculum durch den Einfluss besonders von Felix Klein in diesen Zeiten geändert hatte (Kleinsche Reform). Etwa im Jahr 1925 wurde die Differentialrechnung als Schulstoff etabliert. $\mathrm{Zu}$ Anfang der Rede konstatierte Toeplitz die verfahrene Situation mit den Worten:

Das ist das Bild eines völligen Auseinanderlebens der beiden Instanzen in allen ihren Organen.

Er ging dabei von der damaligen Zahl von $95 \%$ an Studierenden aus, die einmal in den Schuldienst gehen würden. Bis auf diese Beschreibung ähnelt die Sachlage unserer heutigen Zeit. Toeplitz konzentriert sich dann auf die Hochschuldidaktik und bringt sein Anliegen auf den Punkt, nachdem er von den Schwierigkeiten in Kleins Vorlesung „Elementarmathematik vom höheren Standpunkte aus” berichtet 
hat [38, S. 3]:

Felix Klein hat eine Vorlesung über Elementarmathematik vom höheren Standpunkt geschaffen, die zweifellos eine große Wirkung ausgeübt hat. Indessen hat dieser Versuch wenig Nachahmung durch andere Dozenten gefunden, und mit Recht ... so erscheint hier eine Materie von dem übrigen Stoff der Universitätsmathematik losgelöst und für sich behandelt: die Elementarmathematik und was stofflich mit ihr verwandt ist ... Und gerade ein solcher Versuch kann nicht in die Tiefe wirken. Aus dem methodischen Rahmen der Algebra herausgerissen ... Der Ausgleich zwischen Stoff und Methode in den heutigen Universitätsvorlesungen ist im Prinzip nicht der richtige. In diesem Ausgleich sehe ich den Schlüssel zur Lösung des ganzen Aufgabenkomplexes, der von der Leitung des Kongresses mit dem Wort von der „Spannung” so trefflich gekennzeichnet worden ist.

In diesen Bemerkungen zu Felix Klein wird wieder deutlich, wie Toeplitz im Begriff ist, sich von seinem Vorbild zu lösen. Neben der Kritik ist dies besonders in seinem viel weitergehenden inhaltlichen Ansatz zu spüren. Man kann argumentieren, dass Felix Klein einen Standpunkt hatte, der aufgrund seiner Rolle in erster Linie wissenschaftspolitisch angelegt war und Toeplitz sich bemühte, die Praxis der Ausbildung mit Leben zu füllen. Die Verschiedenheit der Personen Toeplitz und Klein wird hier jedenfalls deutlich.

Man muss wissen, dass es zur Zeit dieser Rede an manchen Universitäten eine große Anzahl von Hörern gab. Behnke gibt in [3, S. 5] an, dass es in Bonn 1928-1930 mehrere hundert Mathematikstudenten gab, und die Anfängervorlesung 200 Hörer hatte. Dies nimmt Toeplitz zum Anlass, im Abschnitt A auf die diesbezüglichen Probleme einzugehen, indem er intensiv das Prüfungswesen bespricht. Interessanter sind seine Bemerkungen zu seinem Übungsbetrieb, der ihm sehr am Herzen lag und der unserem heutigen ähnlich gewesen sein muss. Im Hinblick auf die Abbrecherquote ist ihm das Eingehen auf den einzelnen Studierenden sehr wichtig, und er erklärt im Detail seine Bonner Versuche, das Individuum zu fördern. Die intensive Betreuung der Studenten war Toeplitz also sehr wichtig, und dies war Teil seines didaktischen Konzepts. Heinrich Behnke geht auf diese Aktivitäten in [3, S. 5] ein:

In der allgemeinen akademischen Verantwortungs- und Hilflosigkeit fühlt er die Verantwortung für die allzu große Zahl seiner Hörer. Das müßte einmal zum Verderben der Schulen und ihrer Lehrer ausschlagen, wenn man nicht die weniger geeigneten zeitig aus den Hörsälen entfernte. Mit größter Umsicht machte er sich sogleich an die Aufgabe, wenigstens in seinem Felde den schwächeren Studenten zeitig und in einer warmherzig sorgenden Art den Weg zu versperren. Nie las er Vorlesungen ohne Übungen. Dort achtete er genau auf die Leistungen der Teilnehmer. Er kannte die geistigen Fähigkeiten seiner Studenten im einzelnen. So konnte bei ihm nicht leicht eine Arbeit unter fremden Namen laufen, weil er in Ansatz, Ausdruck und Schrift die Eigenarten seiner 200 Hörer kannte und sie immer wieder im Kreise seiner Mitarbeiter besprach. Die Begabtesten aber aus der großen Schar sammelte er zu vielen Sonderbesprechungen um sich. In völlig ungezwungenem und kameradschaftlichen Umgange, der auch auf ihn durch viele Anregungen zurückstrahlte, bemühte er sich, ihre fachliche und allgemeine geistige Entwicklung wesentlich zu fördern. Wenn man ihn aufsuchte, fand man ihn meistens in diesem geistig und menschlich bewegten Kreise, dem er ebensosehr sorgender Freund wie Lehrer war. 
In diesem Zusammenhang ist auch der Bericht von Gottfried Köthe aus [4, S. 10] interessant:

Man traf sich nachmittags bei Toeplitz in seiner schönen Wohnung in der Coblentzer Straße und korrigierte gemeinsam. Wir besprachen anhand der ersten 10 Hefte die möglichen Lösungsmethoden; wir wußten, welche der Studenten zu originellen Gedanken fähig waren und kamen so recht bald zu einer Einteilung, in die die große Masse der Übungen dann leichter einzuordnen war. Toeplitz legte großen Wert auf sehr sorgfältige Durchsicht. Wir waren zu dritt oder zu viert, ich erinnere mich vor allem an Frau Hagemann und an Helmut Ulm, der gerade an seiner später berühmt gewordenen Dissertation zu arbeiten begann. Da es meist über 100 Hefte waren, saßen wir oft bis 9 Uhr abends beisammen, zwischendurch brachte Frau Toeplitz Berge von belegten Brötchen herein.

In Teil B seiner Rede geht Toeplitz auf die Infinitesimalrechnung in der Schule und ihre didaktischen Probleme ein. Er schreibt [38, S. 15]:

Die ganze Schwierigkeit mit der Infinitesimalrechnung auf der Schule ist dadurch entstanden, daß man sie eingeführt hat, ehe man das didaktische Problem gelöst oder auch nur ernsthaft angegriffen hatte, das eben hier aufgeworfen worden ist. Es darf nicht verheimlicht werden, daß es zur Zeit in der Hauptsache noch ungelöst ist. Davon, ob es gelingt, es zu lösen, davon, ob man es überhaupt mit voller Kraft vornimmt, wird es abhängen, ob die Infinitesimalrechnung auf der Schule die Stelle sich für immer erobert, die sie soeben zu besetzen begonnen hat. Gelingt die Lösung nicht, so wird die Infinitesimalrechnung in zwei Dezennien ebenso unrühmlich von der Schule verschwinden, wie heute die Dreiecksaufgaben verschwunden sind.

Die Rede endet mit einem Appell, das Methodische zu ändern, nicht nur das Stoffliche. Mir scheint, dass die damaligen Probleme der Schnittstelle bis heute ungelöst sind, auch wenn es dazu bis heute viele Ansätze gab, womit sich auch die Semesterberichte immer wieder befasst haben.

\section{DAS MATHEMATISChe WeRK VON TOEPLitz}

Zum mathematischen (Euvre von Otto Toeplitz gibt es Übersichtsartikel von Peter Lax in [5], Jean Dieudonné in [8] und Gottfried Köthe [8, 15]. Toeplitz hat bei David Hilbert den Höhepunkt von Hilberts Arbeiten über Integralgleichungen miterlebt. Später hat er zusammen mit Ernst Hellinger einen Beitrag für die Enzyklopädie der mathematischen Wissenschaften geschreiben, in dem die Hauptergebnisse dieser Theorie zusammengefasst sind [36]. Er selbst konzentrierte sich dabei auf die algebraischen Aspekte der Theorie. Toeplitz hat in den Jahren danach versucht, viele endlich-dimensionale algebraische Eigenschaften, wie das Ähnlichkeitsund das Äquivalenzproblem von Matrizen, auf den unendlich-dimensionalen Fall zu verallgemeinern. Zusammen mit Köthe hat er die Theorie der vollkommenen Räume entwickelt, die schließlich in der Theorie der lokalkonvexen und normalen Räume aufgegangen sind. Seine Verhaftung in algebraischen Ausdrucksweisen kam aus der Tradition von Hilbert, den er - wie wir oben beschrieben haben - als „Algebraiker” bezeichnet hat 40, und kann vielleicht auch durch seine frühen Beschäftigungen mit der algebraischen Geometrie erklärt werden. Man kann sagen, dass die Entwicklung der abstrakten Funktionalanalysis Toeplitz irgendwann überholt hatte.

Wir wollen nun einige mathematische Resultate von Toeplitz erklären, mit denen sein Name eng verbunden ist. Ein bekannter, elementarer Satz von Toeplitz ist 
Theorem 0.1 (Hellinger-Toeplitz). Sei T ein selbstadjungierter (symmetrischer) linearer Operator in einem Hilbertraum $H$, dann ist $T$ stetig.

Proof. Sei $\Gamma_{T}$ der Graph von $T$. Wegen des Satzes von der Abgeschlossenheit des Graphen ist zu zeigen, dass jede konvergente Folge $\left(f_{n}, T\left(f_{n}\right)\right)$ in $\Gamma_{T}$ auch ihren Limes in $\Gamma_{T}$ hat. Da $T$ linear ist, können wir annehmen, dass $\left(f_{n}\right)$ eine Nullfolge in $H$ ist. Sei $g:=\lim _{n \rightarrow \infty} T\left(f_{n}\right)$. Wir müssen zeigen, dass $g=0$ gilt. Dazu benutzen wir die Stetigkeit des Skalarproduktes:

$$
\begin{aligned}
\langle g, g\rangle & =\left\langle\lim _{n \rightarrow \infty} T\left(f_{n}\right), g\right\rangle=\lim _{n \rightarrow \infty}\left\langle T\left(f_{n}\right), g\right\rangle=\lim _{n \rightarrow \infty}\left\langle f_{n}, T(g)\right\rangle= \\
& =\left\langle\lim _{n \rightarrow \infty} f_{n}, T(g)\right\rangle=\langle 0, T(g)\rangle=0 .
\end{aligned}
$$

Die Bedeutung des folgenden sogenannten Permanentsatzes von Toeplitz kommt aus der Theorie der Summierbarkeit von konvergenten und divergenten Reihen durch Mittelbildungsverfahren, bei denen man die Folge der Partialsummen $s_{n}=\sum_{k=0}^{n} a_{k}$ einer unendlichen Reihe $\sum_{k=0}^{\infty} a_{k}$ durch die Folge der Ausdrücke

$$
t_{n}=\sum_{q=0}^{\infty} A_{n q} s_{q}
$$

ersetzt, wobei für jedes $n$ nur endlich viele Koeffizienten $A_{n q}$ von Null verschieden sind. Cesàro Summation ist ein bekanntes Beispiel dafür, bei dem

$$
t_{n}=\frac{s_{0}+\cdots+s_{n}}{n+1}
$$

ist. Der Satz von Lipót Fejér, der besagt, dass die Cesàro Summation einer Fourierreihe immer gleichmäßig gegen die Funktion $f$ konvergiert, falls $f$ stetig und periodisch ist, ist eines der wichtigsten Beispiele für solche Summationen. Ein solches Mittelbildungsverfahren heißt regulär, wenn der Grenzwert bei konvergenten Reihen erhalten bleibt.

Theorem 0.2 (Permanentsatz von Toeplitz [49]). Eine solche unendliche Matrix $A=\left(A_{p q}\right)$ definiert ein reguläres Mittelbildungsverfahren genau dann, wenn die folgenden drei Bedingungen erfüllt sind:

- Die Zeilensummen $\sum_{q} A_{p q}$ erfüllen $\lim _{p \rightarrow \infty} \sum_{q} A_{p q}=1$.

- Die Spalten erfüllen $\lim _{p \rightarrow \infty} A_{p q}=0$ für alle $q$.

- Die Summe der Zeilenbeträge $\sum_{q}\left|A_{p q}\right|$ ist beschränkt unabhängig von $p$.

Proof. Dies ist das Hauptergebnis in [49, S. 119].

Gemeinsam mit Felix Hausdorff bewies Toeplitz ein Theorem zum Wertebereich von Operatoren. Sei $T: H \rightarrow H$ ein linearer (nicht notwendig beschränkter) Operator in einem Hilbertraum $H$. Der numerische Wertebereich $W(T)$ von $T$ ist die Menge aller Werte

$$
\{\langle T(x), x\rangle:\|x\|=1\} .
$$

Theorem 0.3 (Satz von Toeplitz-Hausdorff [50]). Der numerische Wertebereich $W(T)$ ist konvex.

Proof. Ein elementarer Beweis findet sich in [9]. 
Wir wollen zum Abschluss erklären, auf welche Weise die sogenannten Toeplitzmatrizen entstehen, die so weit verbreitet in der Mathematik und in Anwendungen sind. Sei dazu $f(\varphi)$ eine reelle, periodische Funktion mit Periode $2 \pi$ und

$$
\sum_{n \in \mathbb{Z}} c_{n} e^{i n \varphi}
$$

die Fourierreihe von $f$. Toeplitz stellt sich in der Arbeit 39] die Frage, wie man die Positivität von $f$ auf $[0,2 \pi]$ durch ein Kriterium an die Koeffizienten $c_{n}$ ausdrücken kann. Ein solches folgt leicht aus folgender Rechnung mit Hilfsvariablen $\xi_{n}(n \in \mathbb{Z})$, welche Peter Lax in [8, S. 87-88] andeutet, unter Verwendung der Orthonormalbasis $\left\{e^{i n \varphi} \mid n \in \mathbb{N}_{0}\right\}$ :

$$
\begin{aligned}
\int_{0}^{2 \pi}\left|\sum_{n=0}^{N} \xi_{n} e^{i n \varphi}\right|^{2} f(\varphi) d \varphi & =\int_{0}^{2 \pi} \sum_{m, n=0}^{N} \xi_{n} \bar{\xi}_{m} e^{i(n-m) \varphi} \sum_{k \in \mathbb{Z}} c_{k} e^{i k \varphi} d \varphi \\
& =2 \pi \sum_{m, n=0}^{N} c_{m-n} \xi_{n} \bar{\xi}_{m} .
\end{aligned}
$$

Den Ausdruck

$$
\sum_{m, n=0}^{\infty} c_{m-n} \xi_{n} \bar{\xi}_{m}
$$

nennt Toeplitz eine L-Form. Somit ist $f$ positiv genau dann, wenn alle quadratischen Formen $\sum_{m, n=0}^{N} c_{m-n} \xi_{n} \bar{\xi}_{m}$ positiv definit sind, d.h. für alle $N$ deren Determinanten positiv sind. Die symmetrische Matrix zu einer solchen Form hat die Gestalt, die man Toeplitzmatrix nennt,

$$
\left|\begin{array}{lllllll}
c_{0} & c_{1} & c_{2} & c_{3} & \ldots & \ldots & \ldots \\
c_{-1} & c_{0} & c_{1} & c_{2} & c_{3} & \ldots & \ldots \\
c_{-2} & c_{-1} & c_{0} & c_{1} & c_{2} & c_{3} & \ldots \\
c_{-3} & c_{-2} & c_{-1} & c_{0} & c_{1} & c_{2} & \ldots \\
\vdots & \ddots & \ddots & \ddots & \ddots & \ddots & \ddots
\end{array}\right|
$$

mit Einträgen $c_{i j}=c_{j-i}$, die nur von $j-i$ abhängen, also auf den Diagonalen konstant sind. Toeplitz zeigt weiterhin, dass der Operator, der durch eine (reguläre) L-Form gegeben wird, als Spektrum genau die Funktionswerte der zu der L-Form zugeordneten analytischen Funktion

$$
\sum_{n \in \mathbb{Z}} c_{n} z^{n}
$$

auf dem Einheitskreis besitzt, siehe [39, Satz 5].

Dank: Ich danke Hans Niels Jahnke, Sebastian Lange, Martin Mattheis, Astrid Mehmel, Sieglinde Müller-Stach, David Rowe, Daniel Schneider, Jörn Steuding, Duco van Straten, Renate Tobies und Klaus Volkert für Diskussionen, Verbesserungen und Hinweise, der Universitätsbibliothek Bonn für die Digitalisierung des Nachlasses sowie die Bonner Mathematischen Schriften und der Universitätsbibliothek Göttingen für die Kopien der Briefe von Toeplitz an Hilbert.

\section{LITERATUR}

[1] Birgit Bergmann und Moritz Epple (Hg.): Jüdische Mathematiker in der deutschsprachigen akademischen Kultur, Springer Verlag (2009).

[2] Heinrich Behnke: Semesterberichte, Vandenhoeck und Ruprecht (1978).

[3] Heinrich Behnke: Otto Toeplitz zum Gedächtnis - Der Mensch und Lehrer, Jahresbericht der DMV, Band 66, 1-8 (1963).

[4] Sonderband Otto Toeplitz 1881-1940, Bonner Math. Schriften Band 143 (1982). 
[5] Stefan Hildebrandt und Peter Lax: Otto Toeplitz, Bonner Math. Schriften Band 319 (1999).

[6] Harald Boehme: Oskar Becker, Bryson und Eudoxos, Mathematische Semesterberichte, Heft 60/1, 85-104 (2012).

[7] Max Born: Obituary Prof. Otto Toeplitz, Nature Band 145, 617 (1940).

[8] Israel Gohberg (ed.): Toeplitz Centennial, Birkhäuser Advances and Applications, Vol. 4 (1982).

[9] Karl Gustafson: The Toeplitz-Hausdorff theorem for linear operators, Proceedings of the American Mathematical Society, 203 (1970).

[10] Uta Hartmann: Heinrich Behnke, Dissertation, Peter Lang Verlag (2009).

[11] Uta Hartmann: Heinrich Behnke und die Entwicklung der Semesterberichte bis Anfang der 1950er Jahre, Math. Semesterberichte Band 55, Heft 1, 69-86 (2008).

[12] Uta Hartmann: Über den Briefwechsel zwischen Karl Jaspers und Heinrich Behnke, Berichte zur Wissenschaftsgeschichte 30, 33-47 (2007).

[13] Marie und Paul Ernst Kahle: Was hätten Sie getan ? Die Flucht der Familie Kahle aus Nazi-Deutschland / Die Universität Bonn vor und während der Nazi-Zeit, Bouvier Verlag (1998).

[14] Felix Klein: Vorlesungen über die Entwicklung der Mathematik im 19. Jahrhundert, 2 Bände, Springer Verlag (1926).

[15] Gottfried Köthe: Otto Toeplitz zum Gedächtnis - Das wissenschaftliche Werk, Jahresbericht der DMV, Band 66, 8-16 (1963).

[16] Sebastian Lange: Otto Toeplitz: Seine Interessen in der Didaktik und der Geschichte der Mathematik, Staatsexamensarbeit Mainz (2013).

[17] Ernst Mach: Die Mechanik in ihrer Entwicklung: historisch-kritisch dargestellt, Brockhaus Verlag, Leipzig, 1883.

[18] Martin Mattheis, Gerd Schubring und Renate Tobies: Felix Klein und die Berliner Schulkonferenz des Jahres 1900, Der Mathematik-Unterricht, Jahrgang 46, Heft 3 (2000).

[19] Erwin Neuenschwander: Der Nachlass von Erich Bessel-Hagen im Archiv der Universität Bonn, Historia Mathematica, Vol. 20, 382-414 (1993).

[20] Quellen und Studien zur Geschichte der Mathematik, Astronomie und Physik, herausgegeben von Otto Neugebauer, Julius Stenzel und Otto Toeplitz, Springer Verlag.

[21] Constance Reid: Courant, Springer Verlag 1979.

[22] Constance Reid: Hilbert, Springer Verlag 1970.

[23] Dora Reimann: Historische Studie über Ernst Machs Darstellung des Hebelgesetzes, Quellen und Studien zur Geschichte der Mathematik, Astronomie und Physik B: Studien, Band 3, Springer Verlag, 554-592 (1936).

[24] Volker Remmert: Ungleiche Partner in der Mathematik im „Dritten Reich”: Heinrich Behnke und Wilhelm Süß, Math. Semesterberichte, Band 49, 11-27 (2002).

[25] David Rowe: Felix Klein, Adolf Hurwitz, and the "jewish question" in german academia, Years Ago, Math. Intelligencer, Vol. 29 No.2, 18-30 (2007).

[26] Daniel Schneider: Oskar Becker - Untersuchungen zur antiken griechischen Mathematik und sein Standpunkt im Grundlagenstreit des frühen 20. Jahrhunderts, Staatsexamensarbeit Mainz (2013).

[27] Gerd Schubring: Das genetische Prinzip in der Mathematikdidaktik, Klett-Cotta, Stuttgart 1978.

[28] Carl Ludwig Siegel: Zur Geschichte des Frankfurter Mathematischen Seminars, Frankfurter Universitätsreden, Heft Nr. 36 (1964), Nachdruck in den Gesammelten Abhandlungen von Siegel, Band 3, Springer Verlag, 462-474 (1966).

[29] Reinhard Siegmund-Schultze: Mathematiker auf der Flucht vor Hitler, Dokumente zur Geschichte der Mathematik, Band 10, Vieweg Verlag (1998),

[30] Walter Stein: Der Begriff des Schwerpunktes bei Archimedes, Quellen und Studien zur Geschichte der Mathematik, Astronomie und Physik B: Studien, Band 1, Springer Verlag, 221-244 (1931).

[31] Ernst Steinitz: Algebraische Theorie der Körper, Crelle Journal, Band 137, S. 167-309 (1910).

[32] Julius Stenzel: Zahl und Gestalt bei Platon und Aristoteles, Teubner Verlag, Leipzig (1924).

[33] Peter Thullen: Erinnerungsbericht für meine Kinder, in: Exil 20, 44-57 (2000).

[34] Otto Toeplitz: Die Entwicklung der Infinitesimalrechnung, Erster Band, Wissenschaftliche Buchgesellschaft (1972).

[35] Otto Toeplitz und Hans Rademacher: Von Zahlen und Figuren, Springer Verlag Heidelberg (1930).

[36] Ernst Hellinger und Otto Toeplitz: Integralgleichungen und Gleichungen mit unendlich vielen Unbekannten, Enzyklopädie der math. Wissenschaften. Band III, B.G. Teubner, Leipzig (1927). 
[37] Otto Toeplitz: Das Problem der Universitätsvorlesungen über Infinitesimalrechnung und ihrer Abgrenzung gegenüber der Infinitesimalrechnung an den höheren Schulen, Jahresbericht der DMV Band 36, 88-100 (1927).

[38] Otto Toeplitz: Die Spannungen zwischen den Aufgaben und Zielen der Mathematik an der Hochschule und an der höheren Schule, Schriften des deutschen Ausschusses für den mathematischen und naturwissenschaftlichen Unterricht, Heft 10 (1928).

[39] Otto Toeplitz: Zur Theorie der quadratischen und bilinearen Formen von unendlich vielen Veränderlichen, 1. Teil: Theorie der L-Formen, Math. Annalen 70, 351-176 (1911)

[40] Otto Toeplitz: Der Algebraiker Hilbert, Die Naturwissenschaften Nr. 4, 73-77 (1922).

[41] Otto Toeplitz: Bericht über die Kieler mathematischen Referendarkolloquien, Deutsches Philologenblatt, Heft 12, 182-184 (1928).

[42] Otto Toeplitz: Die linearen vollkommenen Räume der Funktionentheorie (posthum herausgegeben von G. Köthe), Comm. Math. Helv. 23, 222-242 (1949).

[43] Otto Toeplitz: Die Zukunft unserer jungen Generation. Was macht der jüdische Abiturient ? C-V-Zeitung (1937).

[44] Otto Toeplitz: Nachlass auf Mikrofilm, UB Bonn.

[45] Otto Toeplitz: Mathematik und Antike, In: Die Antike I, de Gruyter Verlag, 175-203 (1925).

[46] Otto Toeplitz: Das Verhältnis von Mathematik und Ideenlehre bei Plato, Quellen und Studien zur Geschichte der Mathematik, Astronomie und Physik B: Studien, Band 1, Springer Verlag, 3-33 (1931).

[47] Otto Toeplitz: Die mathematische Epinomisstelle, Quellen und Studien zur Geschichte der Mathematik, Astronomie und Physik B: Studien, Band 2, Springer Verlag, 334-346 (1933).

[48] Otto Toeplitz: Briefe Toeplitz an Hilbert 1908-1934, in SUB Gött. Cod. Ms. D. Hilbert 408, 16 Blätter.

[49] Otto Toeplitz: Über allgemeine lineare Mittelbildungen, Prac. math.-fiz. 22, 113-119 (1911).

[50] Otto Toeplitz: Das algebraische Analogon zu einem Satz von Fejér, Math. Zeitschrift, 187197 (1918).

[51] Uri Toeplitz: Und Worte reichen nicht, Hartung-Gorre Verlag Konstanz (2000).

[52] Eva Wohl: So einfach liegen die Dinge nicht, Edition Lempertz (2004).

E-mail address: mueller-stach@uni-mainz.de

Johannes Gutenberg-Universität Mainz 